\title{
Gravitational-Wave Astronomy Still in Its Infancy
}

\author{
Gravitational-wave detectors have barely scratched the surface of the \\ treasure trove of discoveries that they could produce.
}

\author{
By B. S. Sathyaprakash and Matthew Evans
}

S eptember 14, 2015 was a momentous day for physics. That day researchers at the Laser Interferometer Gravitational-Wave Observatory (LIGO) made the first detection of gravitational waves, spotting the merger of two black holes (see Viewpoint: The First Sounds of Merging Black Holes) [1]. The observation, known as GW150914, finally verified that these waves exist-a key prediction of Einstein's century-old general theory of relativity (GR). It also brought to fruition a quest that began with experiments in the 1960s [2-4].

Two years later, on August 17, 2017, LIGO and its sister experiment Virgo made another landmark detection-known as

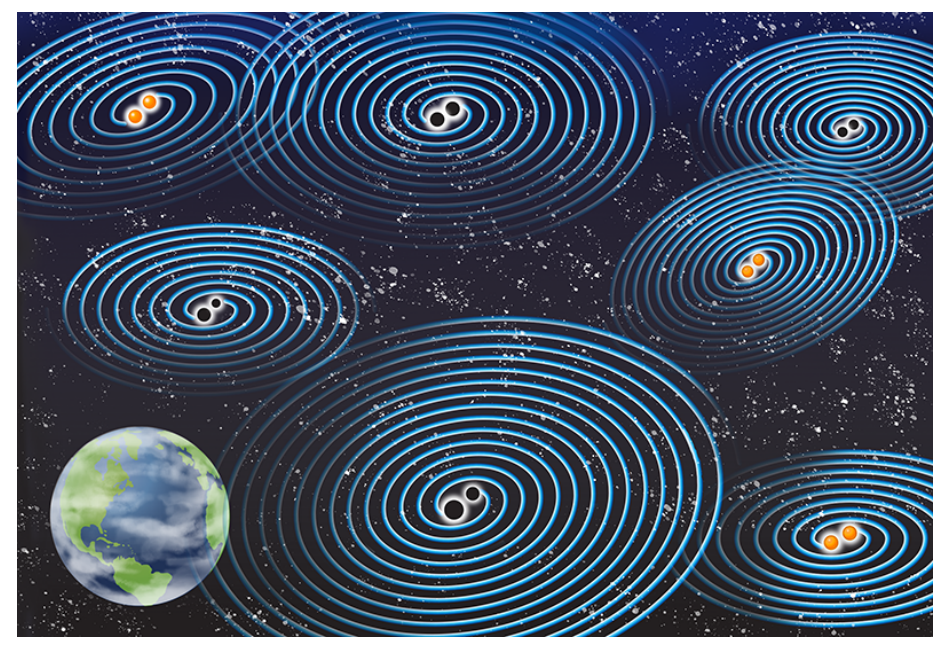

GW170817-when they captured the inspiral and coalescence of a pair of neutron stars (see Viewpoint: Neutron Star Merger Seen and Heard) [5]. The collision produced fireworks across the electromagnetic spectrum that were imaged by telescopes around the world, ushering in the new era of "multimessenger" astronomy with gravitational waves $[6,7]$.

In the four years since that second detection, LIGO and Virgo have provided a treasure trove of gravitational-wave data that has transformed our understanding of black holes and neutron stars [8-10]. Yet this data is only the beginning. New results published today (see Viewpoint: A Heavyweight Merger and Viewpoint: A Lopsided Merger) continue LIGO and Virgo's path of spectacular discoveries that we hope will extend with future observatories $[10,11]$. Together, these facilities will tackle some of the biggest outstanding problems in physics and astronomy.

\section{A New Era in Fundamental Physics \& Astronomy}

LIGO and Virgo were built to observe the gravitational waves emitted from the merger of compact binaries, such as two black holes or two neutron stars. And these types of mergers are the only events that the detectors have confidently spotted in the first two observing runs. These detections have allowed scientists to make significant astrophysical findings, some of which have shaken established wisdom.

Figure 1: With future gravitational-wave observatories, researchers could detect significantly more mergers of black holes and neutron stars. These detections will allow physicists to continue LIGO and Virgo's path of spectacular discoveries.

Credit: APS/Carin Cain

One of the first of those findings is that gravitational waves travel at the speed of light $[6,12,13]$. This result, while consistent with GR, poses a challenge for dark-energy-inspired theories of gravity because those theories require gravitational waves to travel at slower speeds [14]. Another field-shaking advance was the observation that binary neutron star mergers are progenitors of short, hard, gamma-ray bursts, resolving a 


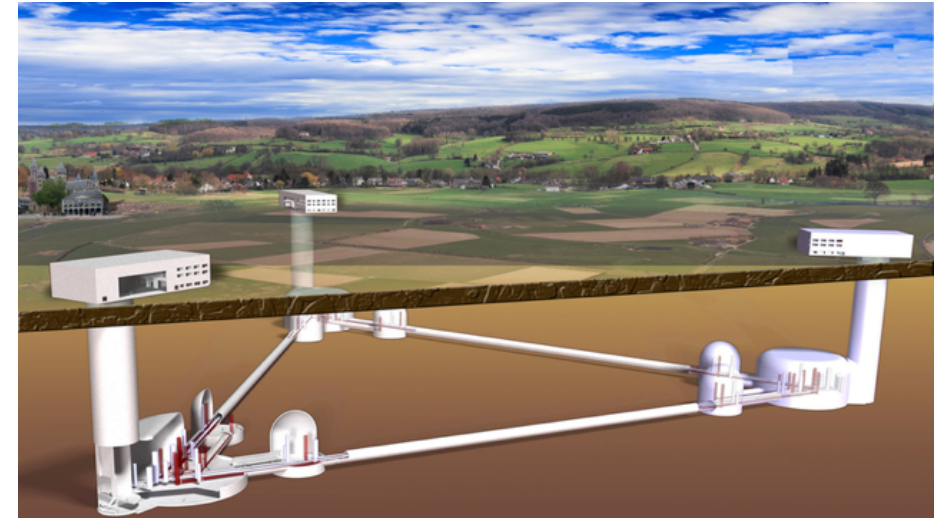

Figure 2: Artist's conception of the Einstein Telescope. The proposed underground detector will consist of six V-shaped, interferometers, laid out in an equilateral triangle with sides that are $10 \mathrm{~km}$ in length.

Credit: ET Design Study team, ET-0106C-10

decades-old puzzle on the origin of these transient high-energy astrophysical events [6]. And then, as announced today, LIGO and Virgo spotted a black hole that lies in the so-called pulsational pair-instability mass gap where no black holes were expected to form [15]. Thus, this detection brings into question current astrophysical models that predict how big black holes form and the environments in which they form.

Taken together these advances solve old questions, challenge what we thought we knew about fundamental processes in stellar evolution, and cast doubts on proposed solutions to current problems in observational astrophysics.

\section{Observatories of the Future}

Over the next few years planned upgrades to LIGO and to Virgo should yield a factor of 5-10 increase in the detection rate of compact binary mergers. The turning on of other gravitational-wave detectors could also increase this rate. KAGRA in Japan-the first gravitational-wave detector in Asia, and the first detector to be built underground-recently began operations. LIGO and Virgo will also soon be joined by LIGO-India, whose construction began this year. (LIGO-India is using the instrumentation developed by LIGO). It is hoped that LIGO-India will be operational after 2025.

Other upgrades and facilities are also being discussed. For example, an upgrade at the existing LIGO sites, named LIGO-Voyager, aims to enhance the sensitivity of Advanced LIGO, leading to a factor of 8 increase in volume coverage and a similar increase in detection rate [16]. There is also the European Einstein Telescope (ET), which was initially conceived in 2008 and is currently at an advanced stage of design (Fig. 2). The ET project is a proposal for an underground observatory that houses three $\mathrm{V}$-shaped detectors at the corners of an equilateral triangle with $10-\mathrm{km}$-long sides [17]. And then there are ideas for Cosmic Explorer in the US, whose arms would be 10 times the length of LIGO's (Fig. 3), and NEMO in Australia, which would target postmerger binary-neutron-star signals [18]. Together, these new facilities will be able to detect events right out to the very edge of the observable Universe. They will also register nearby signals with higher fidelity than currently possible.

More facilities would also help in pinpointing the exact spot from which the gravitational waves emanated. With KAGRA and LIGO-India up and running, researchers could record a "quintuple hit"-the same waves deforming all five detectors. That would enable astronomers to more easily watch the mergers with conventional telescopes, reducing the sky area that they would need to survey by a factor of 4 (see Focus: Possible Flare from Black Hole Merger). These multimessenger observations could reveal significantly more information about the nature and behavior of the sources than can be gleaned from gravitational-wave detectors alone.

\section{Questions for Next-Generation GW Astronomy}

As new observatories, such as the ET and Cosmic Explorer, come online, gravitational-wave observatories will leap from monitoring only the nearby Universe to surveying the entire Universe for black hole mergers. This increase will allow the detectors to peer further back in history and capture black hole and neutron star mergers from epochs when star formation was still in its infancy (Fig. 4).

It is difficult to overstate the discoveries this advance could allow and the potential it offers for unravelling outstanding mysteries of the Universe, as well as for uncovering new physics and astronomical phenomena (Fig. 5). For example, future generations of ground-based gravitational-wave observatories may allow researchers to determine the equation-of-state of the highest density matter, to detect dark matter around black 


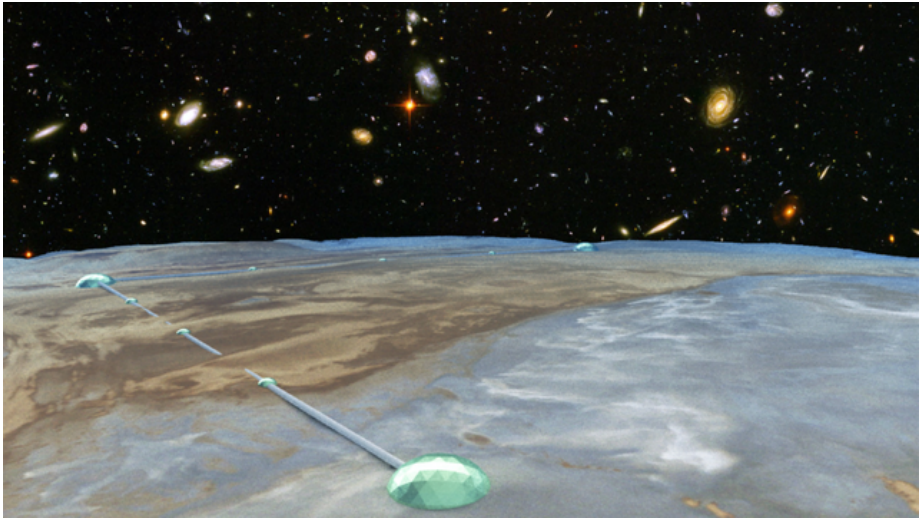

Figure 3: Artist's conception of the Cosmic Explorer detector. The arms of this detector would be $40 \mathrm{~km}$ in length.

Credit: Evan Hall/Massachusetts Institute of Technology; B. S. Sathyaprakash/Pennsylvania State University

holes, and to test modified theories of gravity. We will now describe these opportunities in detail.

Extreme gravity \& fundamental physics: Gravitational waves emanate from regions of spacetime with strong gravity and large spacetime curvature and carry with them uncorrupted information about their sources. Imprinted in the signal are the nature of the source, its physical characteristics (such as the masses of the colliding objects), and the properties of the environment in which the source resides.

Detections of sources with the new observatories could put GR to its most stringent tests to date and could help explore potential violations of the theory in strong fields. For example, the observations could reveal new particles and fields that breach the strong equivalence principle, which, loosely speaking, predicts that freefall accurately simulates zero-gravity conditions in all inertial reference frames. Researchers could also discover violations of the Lorentz invariance (a fundamental symmetry in relativity) or detect polarizations of gravitational waves (the characteristic pattern of the wave's distortion of spacetime) that aren't predicted by GR. We might also infer signatures of quantum gravity. For example, some theories of quantum gravity predict pseudoscalar configurations of gravitational waves that violate parity (a fundamental symmetry that says that the physics of a system and its mirror image should be the same) [19], while others

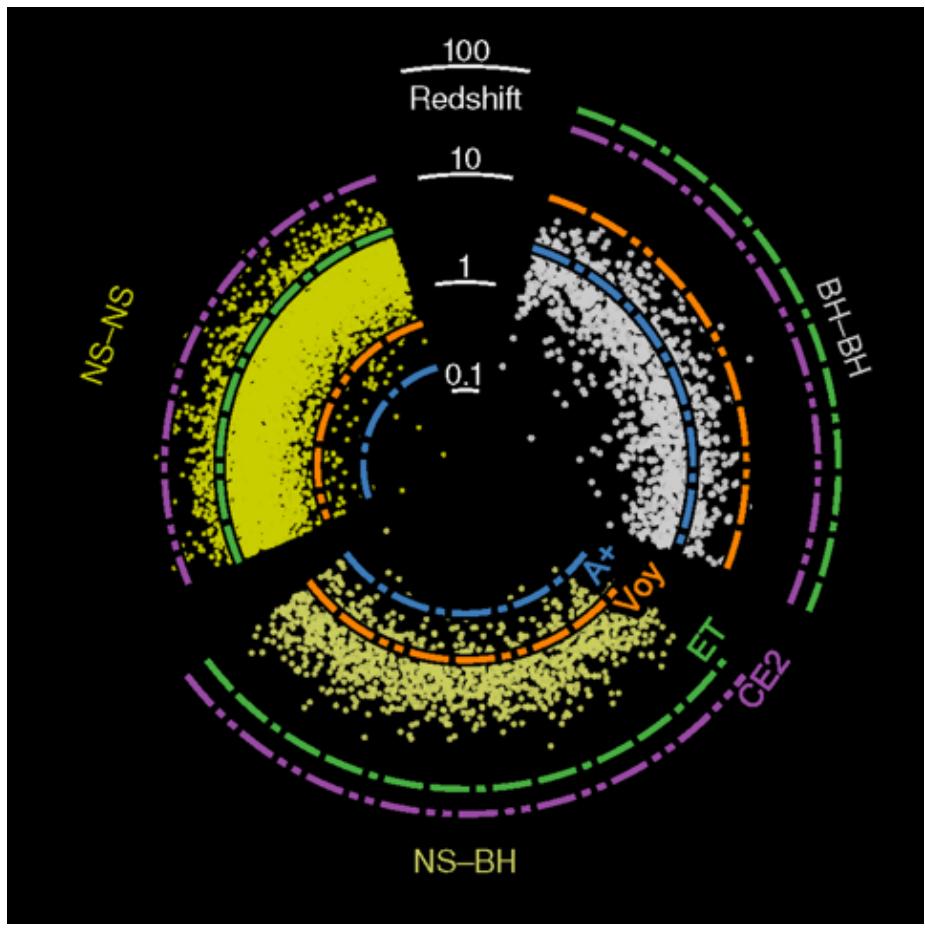

Figure 4: Next-generation observatories will allow researchers to observe binary mergers from the dark ages when the Universe was only a few hundred million years old. The image shows the redshift reach of future observatories, including LIGO Voyager (Voy, orange), the Einstein Telescope (ET, green), and Cosmic Explorer (CE2, purple). The three sections of the images show the redshift for binary neutron star collisions (left upper third), for neutron star-black hole mergers (bottom third), and for binary black hole mergers (right upper third).

Credit: Evan Hall and Salvatore Vitale/Massachusetts Institute of Technology

predict birefringent gravitational waves [20]. Next-generation observatories might also detect the ultralight bosonic fields proposed in certain extensions of the standard model [21]. The evidence for these fields should come from the orbital dynamics of black hole binaries or from the spin properties of black holes.

Extreme matter \& extreme environs: Other astrophysical systems physicists could explore with new detectors are neutron stars, which are the densest objects in the Universe and which have magnetic fields up to billions of tesla in magnitude. Six decades after their discovery, we still don't fully understand the equation of state of the cores of these stars, and we don't know the origin 
of their large magnetic fields. These fields could deform a neutron star causing it to emit gravitational waves, something that future observatories could uncover.

As two neutron stars in a binary spiral in and merge, they subject each other to tidal fields. The state of matter of each star's core determines the magnitude of its tidal deformation, information that should be imprinted in the gravitational waves they emit. Furthermore, the merger of the stars could leave behind a short-lived, hypermassive neutron star, an object that forms in the aftermath of a binary neutron star merger and, for some tens of milliseconds, is supported by rotation against collapse due to its own gravity. Gravitational radiation emitted from this hypermassive neutron star could also reveal unknown physics about the state of ultrahigh-density matter and whether this matter is composed of a quark-gluon plasma.

The electromagnetic follow-up of gravitational-wave observations will offer opportunities to shed light on the origin of $r$-process elements, which form when a nucleus grows larger by rapidly capturing multiple neutrons (see Viewpoint: Out of the Neutron Star Rubble Comes Gold). Finding the origin of heavy elements in the Universe has been a long-standing problem. The electromagnetic observation of GW170817 provided the first irrefutable evidence that binary neutron star mergers produce lanthanides and other heavy elements. But significantly more observations are required to confirm if the mergers alone explain the abundance of heavy elements in the Universe or if other production channels are necessary.

Black holes from the edge of the Universe: Next-generation observatories will chart a complete census of stellar-mass black holes from the present time up until the Universe was just a few hundred million years old and was assembling its first stars. This census will provide key information on the sizes of black holes and allow researchers to uncover how these objects form and grow.

There is increasing evidence that massive black holes exist at the centers of all galaxies. The masses of these black holes (often referred to as supermassive black holes) range from $10^{5}$ to $10^{10}$ times that of the Sun and their size is largely correlated with that of the galaxy. But we don't know how these black holes came to be or how they got so huge. The so-called hierarchical merger model posits that these massive black

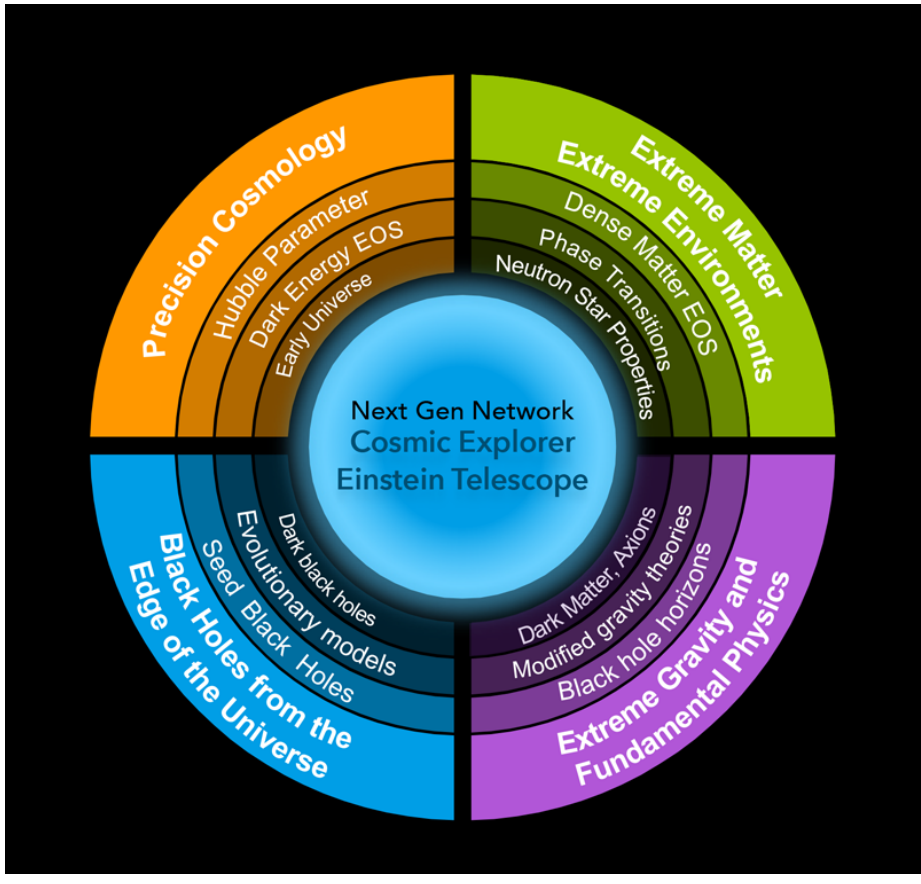

Figure 5: Future ground-based gravitational-wave observatories will tackle fundamental questions on the nature of matter and the nature of spacetime. The dial in the image summarizes the main science themes that next-generation observatories will be able to explore.

Credit: B. S. Sathyaprakash/Pennsylvania State University

holes were initially seeded by heavy stellar-mass black holes, which then merged into larger black holes [22]. An alternative model suggests that massive black holes were seeded by the direct collapse of massive gas clouds [23]. An intriguing third theory speculates that massive black holes formed in the primordial Universe and led to the collapse of dark matter and baryons, triggering the formation of galaxies [24]. The LIGO and Virgo data are so far insufficient to confirm any of these models, but the additional information from future observatories, including the masses of the first black holes in the Universe and the rate at which mergers occur, could identify the origin of supermassive black holes.

Cosmology and the early history of the Universe: Big bang cosmology is largely consistent with GR, but the accelerated expansion of the Universe in its recent history cannot be explained by Einstein's theory. This problem indicates either a failure of the theory or the presence of an-as yet 
unknown-exotic form of energy, called dark energy. Moreover, the observations on galactic to cosmological scales provide indirect evidence for an exotic form of matter, called dark matter, but we still lack direct evidence for both these things.

Future observatories could help in directly detecting dark matter around black holes and around neutron stars. The presence of dark matter could alter the spin of a black hole. It could also modify the orbital dynamics of binary black holes. Alternatively, dark matter could cause neutron stars to implode, making solar-mass-sized black holes that cannot form in any other way. Moreover, with the detection of a larger population of compact binary mergers, and with follow-up electromagnetic observations of the mergers' redshifts, it will be possible to accurately measure cosmological parameters, such as the Hubble parameter [25], the densities of dark matter and dark energy, and the equation of state of dark energy [26]. These measurements are possible since compact binary mergers are so-called standard sirens whose brightness can be used to infer their distances. And as GR completely determines brightness, no astrophysical modeling is required in such measurements [25]. These results will provide a completely independent and complementary measurement of the dynamics of the Universe.

More sensitive detectors could also pick up so-called stochastic gravitational waves, which are expected to have been produced in the early Universe. As the Universe cooled from its primeval hot and dense state it is believed to have undergone several phase transitions that might have generated a gravitational-wave background signal. Detection of this background would dramatically transform our knowledge of particle physics at energy scales that are inaccessible to terrestrial accelerators. Stochastic gravitational waves are also predicted to have emanated from "cosmic strings," hypothetical 1D topological defects associated with a symmetry-breaking phase transition in the early Universe.

Sources at the frontier of observations: Finally, future observatories could help in understanding the behavior of supernovae, of stellar glitches, and of starquakes, three astrophysical phenomena that are poorly understood. These systems should generate gravitational waves that could be detectable with more sensitive detectors. Multimessenger observations combining gravitational observatories with electromagnetic telescopes and with neutrino observatories, will allow us to probe different aspects of these extreme astrophysical events.

In summary, gravitational-wave astronomy promises to answer key questions in physics and astronomy whose solutions could significantly further our understanding of the Universe. With such massive potential rewards, the science case for building new detectors is extremely compelling.

B. S. Sathyaprakash: Department of Physics, Pennsylvania State University, University Park, PA, USA and School of Physics and Astronomy, Cardiff University, Cardiff, United Kingdom

Matthew Evans: Department of Physics, Massachusetts Institute of Technology, Cambridge, MA, USA

\section{REFERENCES}

1. B. P. Abbott et al. (LIGO Scientific Collaboration and Virgo Collaboration), "Observation of gravitational waves from a binary black hole merger," Phys. Rev. Lett. 116, 061102 (2016).

2. A. Einstein, "Die Feldgleichungen der Gravitation," Sitzungsber. Preuss. Akad. Wiss. Berlin (Math. Phys.) 1915, 844 (1915).

3. A. Einstein, "Über Gravitationswellen," Sitzungsber. Preuss. Akad. Wiss. Berlin (Math. Phys.) 1918, 154 (1918).

4. J. Weber, "Detection and generation of gravitational waves," Phys. Rev. 117, 306 (1960).

5. B. P. Abbott et al. (LIGO Scientific Collaboration and Virgo Collaboration), "GW170817: Observation of gravitational waves from a binary neutron star inspiral," Phys. Rev. Lett. 119, 161101 (2017)

6. B. P. Abbott et al., "Gravitational waves and gamma-rays from a binary neutron star merger: GW170817 and GRB 170817A," Astrophys. J. 848, L13 (2017).

7. B. P. Abbott et al., "Multi-messenger observations of a binary neutron star merger," Astrophys. J. 848, L12 (2017).

8. B. P. Abbott et al. (LIGO Scientific Collaboration and Virgo Collaboration), "Tests of general relativity with the binary black hole signals from the LIGO-Virgo catalog GWTC-1," Phys. Rev. D 100, 104036 (2019).

9. B. P. Abbott et al. (LIGO Scientific Collaboration and Virgo Collaboration), "GW190425: Observation of a compact binary coalescence with total mass $3.4 M_{\odot}$," Astrophys. J. 892, L3 (2020). 
10. R. Abbott et al. (LIGO Scientific Collaboration and Virgo Collaboration), "GW190412: Observation of a binary-black-hole coalescence with asymmetric masses," Phys. Rev. D 102, 043015 (2020).

11. R. Abbott et al. (LIGO Scientific Collaboration and Virgo Collaboration), "GW190521: A binary black hole merger with a total mass of $150 M_{\odot}$, , Phys. Rev. Lett. 125, 101102 (2020).

12. J. M. Ezquiaga and M. Zumalacárregui, "Dark energy in light of multi-messenger gravitational-wave astronomy," Front. Astron. Space Sci. 5 (2018).

13. J. Sakstein and B. Jain, "Implications of the neutron star merger GW170817 for cosmological scalar-tensor theories," Phys. Rev. Lett. 119, 251303 (2017).

14. C. de Rham et al., "Rethinking gravity," New Scientist 247, 31 (2020).

15. Z. Barkat et al., "Dynamics of supernova explosion resulting from pair formation," Phys. Rev. Lett. 18, 379 (1967).

16. R. X. Adhikari et al., "Astrophysical science metrics for next-generation gravitational-wave detectors," Class. Quantum Gravity 36, 245010 (2019).

17. M. Punturo et al., "The Einstein Telescope: A third-generation gravitational wave observatory," Class. Quantum Gravity 27, 194002 (2010).

18. B. P. Abbott et al. (LIGO Scientific Collaboration), "Exploring the sensitivity of next generation gravitational wave detectors," Class. Quantum Gravity 34, 044001 (2017).

19. S. H. Alexander and N. Yunes, "Gravitational wave probes of parity violation in compact binary coalescences," Phys. Rev. D 97, 064033 (2018).

20. S. Alexander and J. Martin, "Birefringent gravitational waves and the consistency check of inflation," Phys. Rev. D 71, 063526 (2005).

21. A. Arvanitaki et al., "String axiverse," Phys. Rev. D 81, 123530 (2010).

22. M. Volonteri et al., "The assembly and merging history of supermassive black holes in hierarchical models of galaxy formation,” Astrophys. J. 582, 559 (2003).

23. M. C. Begelman et al., "Formation of supermassive black holes by direct collapse in pre-galactic haloes," Mon. Not. R. Astron. Soc. 370, 289 (2006).

24. J. García-Bellido, "Massive primordial black holes as dark matter and their detection with gravitational waves," J. Phys: Conf. Ser. 840, 012032 (2017).

25. B. F. Schutz, "Determining the Hubble constant from gravitational wave observations," Nature 323, 310 (1986).

26. B. S. Sathyaprakash et al., "Cosmography with the Einstein Telescope," Class. Quantum Gravity 27, 215006 (2010). 